thrombotic and atherogenic processes contributing to cardiovascular disease.(2) However, the role of NETs in cardiovascular disease in human gout is not known. Objectives: Our objective is to investigate the association between neutrophil activation and cardiovascular risk in gout patients. We hypothesize that neutrophil activation mediates inflammation, as well as activation and damage to endothelial cells, partaking in atherosclerosis development.

Methods: Plasma samples from 75 gout patients participating in the 'Reade gout cohort Amsterdam' were analyzed. Patient data was collected on disease activity, demographics, gout history, comorbidities, medication use and cardiovascular risk assessments. Measurements included anthropometry, vital parameters (RR, $\mathrm{HF}$ ), ECG and lab variables. Levels of NETs, and NET-derived markers (cell-free DNA and peroxidase activity) were analyzed using a MPO-DNA ELISA, as well as fluorimetry. Levels of calprotectin (S100A8/A9) were analyzed by ELISA. Markers of NETosis were related to clinical markers of cardiovascular risk, including BMI, smoking, blood pressure, lipid profile and 10 year risk of cardiovascular mortality (SCORE EU).

Results: No associations were found between markers of cell death (cfDNA and NETs) and cardiovascular risk. However, markers of neutrophil activation, including peroxidase activity correlated with $\mathrm{BMI}(\mathrm{r}=0.31, \mathrm{p}=0.008)$, waist-hip ratio $(r=0.52, p<0.001)$, cholesterol ratio $(r=0.51, p<0.001)$, and triglycerides $(r=0.42$, $\mathrm{p}<0.001)$. These associations were even stronger in patients with chronic, polyarticular gout. Peroxidase activity was associated with the 10 year risk of cardiovascular comorbidity $(r=0.47, p<0.001$, Figure $1 \mathrm{~A})$. Calprotectin levels were elevated in hypertension $(p=0.005)$ and diabetes $(p=0.02)$, with calprotectin levels associating with diabetes independently on $\mathrm{BMI}(\mathrm{OR}=6.2, \mathrm{p}=0.04)$. Finally, we constructed a neutrophil risk score ranging from $0-2$ based on positivity for peroxidase and/or calprotectin to identify patients with a 'neutrophil activation signature'. The neutrophil risk score strongly associated with CVD risk (Figure 1B). Patients with neutrophil activation signature (risk score 1-2) had markedly elevated cardiovascular risk score $(p=0.001)$, with $67.7 \%$ of the patients having high cardiovascular risk, versus $32.3 \%$ of the patients without a neutrophil activation signature $(O R=2.9$, $\mathrm{p}=0.03$ ).

Conclusion: We have demonstrated, for the first time, that neutrophil activation markers are associated with several risk factors of cardiovascular disease, including hyperlipidemia, hypertension and diabetes in a large cohort of gout patients. Furthermore, the presence of a neutrophil activation signature is strongly associated with a 10-year risk of cardiovascular comorbidity.

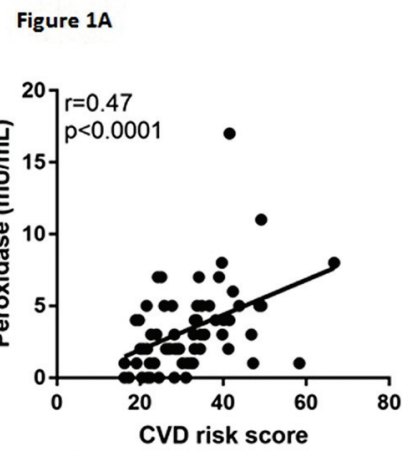

Figure 1B

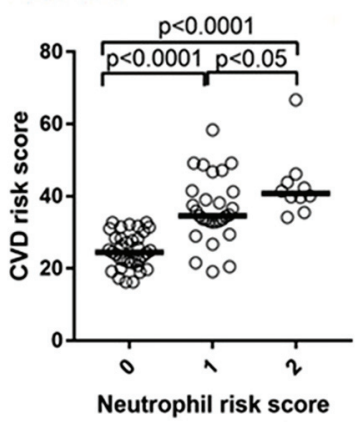

REFERENCES:

[1] Krishnan E. Inflammation, oxidative stress and lipids: the risk triad for atherosclerosis in gout. Rheumatology (Oxford). 2010;49(7):1229-38.

[2] Doring $Y$, et al. Neutrophil Extracellular Traps in Atherosclerosis and Atherothrombosis. Circ Res. 2017;120(4):736-43.

Disclosure of Interests: Daisy Vedder Speakers bureau: Novartis, Martijn Gerritsen Grant/research support from: Grunenthal has sponsored the Reade Cohort, Michael Nurmohamed Grant/research support from: AbbVie, Bristol-Myers
Squibb, Celgene, Eli Lilly, Janssen, Menarini, MSD, Mundipharma, Pfizer, Roche, Sanofi and UCB, Consultant for: AbbVie, Bristol-Myers Squibb, Celgene, Eli Lilly, Janssen, Menarini, MSD, Mundipharma, Pfizer, Roche, Sanofi and UCB, Speak ers bureau: AbbVie, Bristol-Myers Squibb, Celgene, Eli Lilly, Janssen, Menarini, MSD, Mundipharma, Pfizer, Roche, Sanofi and UCB, Ronald van Vollenhoven: None declared, Christian Lood: None declared DOI: 10.1136/annrheumdis-2019-eular.4793

\section{OP0301 \\ C-X-C MOTIF CHEMOKINE 10 (CXCL10) IS A POTENTIAL BIOMARKER OF DISEASE ACTIVITY IN PSORIATIC ARTHRITIS (PSA)}

$\underline{\text { Rohan Machhar }}^{1}$, Sofia Oke ${ }^{1}$, Justine Ye ${ }^{1}$, Fatima Abji ${ }^{1}$, Vinod Chandran ${ }^{1,2,3,4}$. ${ }^{1}$ Krembil Research Institute, University Health Network, Psoriatic Arthritis Program, Centre for Prognosis Studies in the Rheumatic Diseases, Toronto, Canada; ${ }^{2}$ Faculty of Medicine, University of Toronto, Division of Rheumatology, toronto, Canada; ${ }^{3}$ University of Toronto, Department of Laboratory Medicine and Pathobiology, Toronto, Canada; ${ }^{4}$ Memorial University of Newfoundland, Faculty of Medicine, Newfoundland, Canada

Background: Assessment of Psoriatic Arthritis (PsA) disease activity requires detailed clinical evaluation complemented by imaging. Current laboratory markers such as CRP do not accurately reflect PsA disease activity. Identifying soluble biomarkers of PsA disease activity is an important perceived clinical need.

Objectives: We aimed to identify markers that correlates with PsA disease activity and change with treatment.

Methods: Serum samples were obtained from a cohort of patients with PsA not on DMARD treatment assessed clinically every 6 months according to a standard protocol including several measures of disease activity: physician and patient global assessment, tender and swollen joint counts, skin scores (BSA, PASI) and the composite measures- Disease Activity Score for PsA (DAPSA) and PsA Disease Activity Score (PASDAS). In the discovery phase, we mined our clinical and biomarker database that has linked biomarker and disease activity measures and identified markers that potentially reflect PsA disease activity. In verification phase 1 we measured the selected 17 markers in 80 PsA samples. 8 Markers that significantly correlated with disease activity were identified based on Spearman correla tion coefficients. In the verification phase 2 these 8 markers were further assayed in an inception cohort of 80 patients. In this phase, physician global assessment of disease activity (including skin and joints-MDGA) was used as the primary measure of disease activity; other skin, MSK and patient reported measures were considered secondary measures. In verification phase 3 with the hypothesis that markers associated with disease activity would decrease with treatment, the markers that correlated with MDGA where further assayed in 40 patients before and after 6 months of treatment ( 26 biologics and methotrexate, 14 methotrexate) All assays were done using commercially available ELISA.

Results: In verification phase 18 markers of 17 tested were found to be associated with measures of disease activity in 80 PsA patients (mean age 47, males $58 \%$, swollen joint count [SJC] 2, tender joint count [TJC] 3.6, PASI 2.9). In verification phase 22 markers [CXCL10 $(\rho=0.25, \mathrm{p}=0.03)$ and CRP $(\rho=0.26, \mathrm{p}=0.02)$ ] tested in 80 patients (mean age 49, males 54\%, SJC 1.5, TJC 3.3, PASI 5.6) correlated with MDGA, and did not correlate with each other. Three other markers correlated with secondary measures; COMP correlated with PASDAS $(\rho=0.22, \mathrm{p}$ $=0.04)$, MMP3 correlated with PASI $(\rho=0.27, \mathbf{p}=0.01)$ and CPII-C2C ratio correlated with active joints $(\rho=-0.27, p=0.02)$. In verification phase 3 CXCL10 (baseline mean $=246.7$ (standard deviation 190.79) - 6 month 177.7 (163.8)) $(p=0.0008)$ and CRP (18.5 (19.1) - $9.5(8.9)) ;(p=0.002)]$ significantly decreased 6 months post treatment.

Conclusion: CXCL10, a marker previously associated with PSA, may also be a marker of PsA disease activity. Identifying a robust outcome measure for disease activity to conduct biomarker studies in a heterogeneous disease like PsA remains a significant challenge.

Disclosure of Interests: None declared

DOI: 10.1136/annrheumdis-2019-eular.7435

\section{OP0302 MUCOSAL-ASSOCIATED INVARIANT T (MAIT)-CELL- DERIVED IL-17A AND IL-17F PRODUCTION IS IL-23- INDEPENDENT AND BIASED TOWARDS IL-17F}

Suzanne Cole, Catherine Simpson, Remi Okoye, Meryn Griffiths, Dominique Baeten, Stevan Shaw, Ash Maroof. UCB Pharma, Slough, United Kingdom

Background: The requirement for IL-23 in driving IL-17A and IL-17F production in humans is incompletely understood. Preclinical data support IL-17F, together with IL-17A, as a key driver of chronic tissue inflammation. ${ }^{1}$ MAIT cells, an innatelike $T$ cell population, uniformly express ROR $\gamma$ t but only a minority have been shown to produce IL-17A. ${ }^{2}$ Dysregulation in frequency and function of MAIT cells 